\title{
Harumi KINOSHITA
}

\section{Les échanges culturels entre le Japon et la France à l'épreuve de la diplomatie}

\section{Résumé}

L'histoire et l'actualité des échanges culturels entre le Japon et la France, à travers le prêt d'œuvres et d'expositions, méritent d'être examinées de près. En se servant des méthodologies issues de la muséologie, complétées par une approche politique et économique de la question, ce texte analyse le déplacement des œuvres et des expositions entre le Japon et la France. II s'efforce de mettre en évidence les fonctions du prêt des œuvres et des expositions au regard de la diplomatie culturelle d'un pays. En mettant l'accent sur la France, laquelle possède un patrimoine mondialement connu, ce travail montre que le déplacement des œuvres et des expositions est un moyen au service de la diplomatie culturelle.

Mots-clés : déplacement du patrimoine, échanges culturels, diplomatie culturelle.

\begin{abstract}
The history and current reality of cultural exchanges between Japan and France, through the lending of works of art and exhibitions, deserve close examination. Making use of methodologies deriving from a museology, complemented by a political and economic approach to the issue, this text analyze the displacement of works of art and exhibitions between Japan and France. It aims to highlight the function of lending works of art and exhibitions in relation to the cultural diplomacy of a country. By focusing on France, which has world-renowned heritage, this work argues that displacement of works of art and exhibitions is a means of cultural diplomacy.
\end{abstract}

Keywords : displacement of cultural heritage, cultural exchanges, cultural diplomacy. 


\section{Introduction}

Depuis plusieurs années, le public japonais a accès aux chefs-d'œuvre du monde entier. Par exemple, en 1958, les œuvres de Vincent Van Gogh, telles Le Pont de l'Anglais, ont été présentées au Japon dans le cadre de l'exposition Vincent Van Gogh organisée par le Musée Kröler-Müller. Cette exposition a été montrée au Musée national de Tokyo ainsi qu'au Musée municipal des Beaux-Arts de Kyoto. En 1971, La Maja nue et La Maja vêtue de Goya ont été montrées au Musée national d'art occidental et au Musée municipal des Beaux-Arts de Kyoto dans le cadre de l'exposition Goya, organisée par le Musée du Prado, soutenue par le gouvernement espagnol. En 1984, les œuvres de Vermeer, comme La Jeune Fille à la perle, ont voyagé au Japon dans le cadre de l'exposition La Mauritshuis présentée au Musée national d'art occidental, au Musée d'art préfectoral d'Aichi et au Musée d'art préfectoral d'Hokkaido. La présentation de ces chefs-d'œuvre au public japonais a été réalisée grâce au déplacement des collections et des expositions des musées étrangers. On remarque que le public japonais peut bénéficier de ce type d'opération presque chaque année. Cependant, les dispositifs qui les régissent - tels le mécanisme du prêt des œuvres et des expositions - sont méconnus du public.

On peut dire que le Japon est une destination majeure du point de vue du prêt des œuvres et des expositions. Comme nous l'avons vu, les chefs-d'œuvre provenant de grands musées à l'échelle internationale viennent au Japon. Cependant, il faut noter que le musée français, et plus largement la France, est l'un des premiers prêteurs d'œuvres et d'expositions pour le Japon grâce à ses collections prestigieuses. D'ailleurs, l'échange entre le Japon et la France, à travers le prêt d'œuvres et d'expositions, est historiquement remarquable. Rappelons que le Japon est le seul pays qui a accueilli La Vénus de Milo, I'un des trésors majeurs du Louvre. De même, la France est le seul pays auquel le Japon ait confié Kudara Kannon, considéré comme le plus célèbre trésor national du Japon. Ces échanges nous font réfléchir à ce qu'ils signifient : ils peuvent certes sceller l'amitié entre ces deux pays, mais ne sont pas exempts de retombées économiques. Interroger les échanges culturels à partir des prêts d'œuvres et d'expositions revient ainsi à questionner la diplomatie culturelle entre les pays.

Le déplacement des œuvres et des expositions contribue à développer la dimension culturelle mais également la dimension politique et économique. Ce déplacement est en effet un outil de la diplomatie culturelle, outil utilisé par un pays comme la France, lequel possède un patrimoine mondialement connu. L'objectif de cet article est d'analyser la diplomatie culturelle d'un pays comme la France à travers le prêt des œuvres et des expositions. À cette fin, nous commencerons par analyser le patrimoine dans le contexte économique. Ce qui nous conduira à interroger la notion de patrimoine comme outil de 
négociation entre les pays. Enfin, les dernières lignes seront consacrées au déplacement du patrimoine dans le contexte de la diplomatie culturelle.

\section{Le chef-d'œuvre : un outil d'intervention qui impacte l'économie}

Cette première partie sera consacrée à l'examen de deux prêts : le prêt de La Vénus de Milo au Japon en 1964, qui est un prêt exceptionnel dans l'histoire de la muséographie effectué sous la direction du ministère Malraux, et celui de L'Angélus de Millet en 1982. Cette étude nous révélera la raison pour laquelle ces œuvres célèbres, clés de l'attractivité du Musée du Louvre ${ }^{1}$, ont néanmoins été déplacées jusqu'au Japon.

\subsection{Le prêt de La Vénus de Milo au Japon en $1964^{2}$}

La Vénus de Milo a voyagé jusqu'au Japon en 1964. Elle a été exposée au Musée d'art occidental de Tokyo et au Musée municipal des Beaux-Arts de Kyoto. Exposer La Vénus de Milo était un souhait japonais qui relevait du symbole, un symbole fort qui contribuait, comme le fit l'organisation les Jeux Olympiques, à inscrire le Japon au rang des pays développés. II revient au grand journal Asahi Shimbun d'avoir pris l'initiative culturelle, diplomatique et aussi économique de cette exposition. Il fut décidé de la sponsoriser, une décision qui atteste d'un mode de financement spécifique au Japon.

Takio Enna, le chef du département du projet culturel du journal Asahi Shimbun, a adressé une lettre datée du 23 mai 1963 au conservateur du Musée national d'Art moderne, Bernard Dorival, en demandant le prêt de La Vénus de Milo. D'après cette lettre, l'ambition de planifier ce projet exceptionnel et ambitieux est de s'harmoniser avec les Jeux Olympique de Tokyo de 1964. La Vénus de Milo est ici considérée comme l'égale de ce grand événement sportif par son impact sur la psychologie du public et par la signification culturelle qu'elle revêt (AMN, 4CC49).

Le 12 février 1964, l'accord fut conclu entre le gouvernement japonais et le gouvernement français, accord portant sur le prêt de La Vénus de Milo au Japon. L'attestation, écrite par le Directeur des Musées de France, Jean Chatelain, confirme que cette sculpture est prêtée au gouvernement japonais à l'occasion des Jeux Olympiques avec, comme valeur d'assurance, la somme de cent cinquante mille francs (AMN, 4CC49). Le directeur du Musée national de l'Art occidental, Soichi Tominaga, envoie alors une lettre de remerciement datée du 13 janvier 1964 à Jean Chatelain. II conclut cette lettre en souhaitant le

\footnotetext{
${ }^{1}$ L'Angélus de Millet a été transféré au Musée d'Orsay en 1986.

${ }^{2}$ Cette partie est basée sur le chapitre I de la thèse de l'auteur. KINOSHITA Harumi, 2011 : La diffusion culturelle internationale : les enjeux de la politique de prêts d'œuvres et d'expositions du Mnam-Cci (Centre Georges Pompidou) pendant la période 2000-2007, thèse de doctorat, Université d'Avignon et des Pays du Vaucluse.
} 
renforcement des liens entre le Japon et la France dans le domaine des échanges culturels et artistiques (AMN, 4CC49).

Même si le prêt de La Vénus de Milo était en lien avec les Jeux Olympiques et visait à renforcer le prestige national japonais, ce prêt a aussi été envisagé par la France comme un moyen de recevoir une retombée économique, comme l'explique Jean Chatelain, directeur des Musées de France. Ce dernier précise les modalités économiques du prêt dès sa préparation (AMN, 4CC49). Afin de compléter l'accord, il est spécifié que le gouvernement japonais assume tous les frais de publication. Les organisateurs japonais versent à la Réunion des musées nationaux un pourcentage de dix pour cent sur les ventes de photographies et de moulages faites au Japon pendant la durée des deux expositions. De plus, le Japon doit utiliser une copie des films réalisés par la Réunion des musées nationaux et la diffusion de ces films ou émissions de télévision sur les territoires autres que les territoires japonais et français peut être assurée librement par les organisateurs japonais, à charge pour eux de verser à la Réunion des musées nationaux un pourcentage de vingtcinq pour cent sur le montant des droits de reproduction qui leur sont accordés. Les factures qui correspondent au transport de La Vénus de Milo sont établies au nom du journal Asahi Shimbun, les billets d'avion Paris-Tokyo et le retour pour les différents convoyeurs (dix billets au total) sont pris directement en charge par le journal Asahi Shimbun (AMN, $4(C 49)^{3}$.

En termes de fréquentation, la présentation de La Vénus de Milo à Tokyo et à Kyoto a remporté un vif succès comme le montre la file d'attente de quatre kilomètres de long qui emplissait le parc d'Ueno de Tokyo et comme le montre aussi la patience des visiteurs, le dernier jour, devant faire face à une attente de six heures à Kyoto avant de visiter l'exposition (DOC. AGER, Ma0399).

Le nombre total des visiteurs atteint 832382 personnes à Tokyo pendant la période du 8 avril au 15 mai et 891094 personnes à Kyoto pendant la période du 21 mai au 25 juin. Le 10 mai, on comptabilise 35962 visiteurs à Tokyo et, le 21 juin, 52245 visiteurs à Kyoto (DoC. AGER, Ma0399). Le nombre total des visiteurs s'élève à 1723476 sur soixante-quatorze jours. Nous pouvons comparer ce chiffre à celui de l'exposition Art français en 1961, visible pendant cent dix-neuf jours, qui a accueilli 1477388 visiteurs, un chiffre qui représente un record au Japon. La Vénus de Milo a ainsi, on le constate, établi un record dans l'histoire des expositions d'art au Japon (DOC. AGER, Ma0399).

\footnotetext{
${ }^{3}$ Il est aussi précisé que l'ensemble du transport sur le territoire japonais est effectué par camion avec escorte de la police, et que le responsable au Japon de l'ensemble des opérations depuis le débarquement de La Vénus jusqu'à son rembarquement est Monsieur Takio Enna, le directeur du service culturel du journal Asahi Shimbun.
} 
L'impact économique a été également remarquable. Le total de la vente du catalogue s'élève à 431583 exemplaires ; 243671 exemplaires à Tokyo et 187912 exemplaires à Kyoto. Et le total de la vente des souvenirs, comme les reproductions à encadrer par BenriDô et Bijutsu-Shuppan, les cartes postales par Ôtsuka-Kôgei, les moulages par KyotoKagaku, est de 187 millions de yen ; 90 millions de yen à Tokyo et 97 millions de yen à Kyoto. Dix pour cent du total de la vente revient aux royalties et 18741707 de yen sont versés à la France (DOC. AGER, Ma0399).

En remerciement de ce succès, le chef du département du projet culturel du journal Asahi Shimbun, Takio Enna, a adressé un rapport daté du 2 juin 1964 au secrétaire général des Affaires Culturelles Jacques Jaujard. Ce rapport a insisté sur le fait que ce prêt exceptionnel a consolidé l'amitié entre le Japon et la France (DOC. AGER, Ma0399).

Comme nous l'avons vu, le prêt de La Vénus de Milo a un caractère économique lié à un grand événement sportif: les Jeux Olympiques. À l'occasion de ces Jeux Olympiques de 1964, les premiers Jeux Olympiques en Asie, le Japon voulut montrer au monde entier sa reconstruction après la guerre et sa forte croissance économique. Le prêt de La Vénus de Milo peut être considéré comme le symbole de la confiance que la France accorde à la nation japonaise, lui reconnaissant sa maturité et la qualité de ces échanges. Par ailleurs, La Vénus de Milo, elle, a établi le record du nombre de visiteurs dans I'histoire des expositions d'art au Japon et engendra des retombées économiques. Cela montre que les chefsd'œuvre peuvent satisfaire la demande sociale liée à la dimension économique.

\subsection{L'Angélus de Millet au Japon (1982)}

L'exposition L'Angélus de Millet: Tendances du Réalisme en France 1848-1870 a été présentée au Musée national de l'Art occidental de Tokyo du 17 avril au 13 juin 1982. En effet, le public japonais apprécie au plus haut point L'Angélus de Millet, comme l'explique un texte du catalogue de cette exposition (cat. exp. 1982). Dans le même catalogue, Hubert Landais, directeur des Musées de France et président du Conseil International des Musées, souligne l'adoration du public japonais pour cette œuvre qu'il souhaite présenter au Japon. II rend aussi compte des hésitations de la France à prêter cette œuvre, d'une part en raison de sa fragilité et des risques encourus lors de son déplacement, et, d'autre part, en raison d'une possible déception des visiteurs du Louvre en raison de son absence (cat. exp. 1982).

D'après le directeur du Musée national de l'Art occidental, l'offre de la France à prêter L'Angélus de Millet au Japon est venue soudainement (cat. exp. 1982). La France a pris l'initiative de ce prêt à l'occasion d'une visite officielle du président de la République François Mitterrand au Japon prévue en avril 1982. Le Musée national de l'Art occidental a accepté cette offre de la part de France. Il fut décidé de mettre en place une exposition, en 
rapport avec la collection Matsukata ${ }^{4}$, en montrant un mouvement artistique français, le Réalisme, daté de la seconde moitié du XIX siècle. Les artistes présentés dans cette exposition furent Courbet, Corot, Rousseau, Manet et Bazille. Et bien sûr Millet.

Interrogeons-nous à présent sur la raison qui poussa le président Mitterrand à prêter L'Angélus au Japon. En effet, cette visite effectuée par Mitterrand était la première visite d'État d'un président de la République française au Japon. Pour répondre à cette question, il faut se pencher sur la relation politique et économique entre le Japon et la France à la fin des années 1970 et au début des années 1980.

D'un point du vue politique, Valéry Giscard d'Estaing fut président de la République française entre 1974 et 1981. Pendant son mandat, la relation entre la France et le Japon était très distante. On peut dire que la relation entre les deux pays s'était déjà refroidie sous le mandat du président Pompidou 5 . Sur le plan économique, on relève aussi des conflits commerciaux en raison du renforcement de la compétitivité japonaise en termes d'exportations dans le secteur industriel (GEFJ s. d.).

En mai 1981, Mitterrand est élu président de la République française. Les conflits commerciaux entre le Japon et la France ne sont pas pour autant réglés. Cependant, les proches conseillers de Mitterrand étaient japanophiles. La raison pour laquelle le président Mitterrand rend officiellement visite au Japon a dû résoudre quelques problèmes commerciaux et améliorer la relation entre ces deux pays. En effet, les gouvernements japonais et français ont investi, semble-t-il, dans un maximum d'efforts afin de remédier à cette situation comme les articles de journaux le précisent.

\footnotetext{
${ }^{4}$ Kôjirô Matsukata (1866-1950), le troisième fils de Masayoshi Matsukata, est un homme politique de la période Meiji. C'est un homme d'affaires et collectionneur d'art. Il a commencé à rassembler des œuvres d'art à Londres, au milieu de la Première Guerre mondiale. On dit que l'ensemble de sa collection a atteint 10000 pièces, y compris environ 8000 estampes japonaises qui sont maintenant dans la collection du Musée national de Tokyo. Sa passion de collectionner des œuvres d'art est née de sa volonté de construire un musée d'art. Malheureusement, son souhait n'a pas été exhaussé à cause de la crise de 1927 et sa collection, vendue dans une série de ventes aux enchères, a été dispersée. Cependant, il a laissé un grand nombre d'œuvres en Europe. Celles qui sont stockées à Londres ont été détruites par un incendie en 1939, et quelques 400 œuvres furent laissées à Paris sous la protection de Léonce Bénédite, directeur du Musée du Luxembourg, qui les a stockées au Musée Rodin dont il était également le directeur. Sa collection a été mise sous séquestre par le gouvernement français vers la fin de la Seconde Guerre mondiale et elle est devenue bien national de la République française en 1951 dans le cadre du Traité de paix de San Francisco. Pourtant, le gouvernement français a décidé de rendre la majorité des œuvres au gouvernement japonais. Sa collection a été retournée en 1959, ce qui a conduit à l'ouverture du Musée national d'art occidental.

${ }^{5}$ En ce qui concerne la diplomatie japonaise entre 1973 et 1974 et la relation entre le Japon, les États-Unis et l'Europe, voir l'article en japonais rédigé par YАМАМОтО Ken paru dans Nucb Journal of Economics and Information Science [en ligne], vol. 57, $\mathrm{n}^{\circ} 2$, mars. Disponible sur https://www.nucba.ac.jp/en/university/library/journal/nucb/library journal e/vol57 no2/ (consulté le 3 août 2021).
} 
L'article du journal Nihon Keizai Shimbun daté du 17 mars 1982 mentionne que le ministre du Commerce extérieur, Michel Jobert, demande avec insistance au Japon des mesures d'ouverture du marché, cela avant la visite du président Mitterrand (Le journal du soir Nihon Keizai Shimbun, 17 mars 1982, p. 1). Ce ministre insiste sur le fait que la France ne peut pas revenir sur ses mesures protectionnistes si la négociation échoue avant la visite du président Mitterrand (Le journal du soir Nihon Keizai Shimbun, 17 mars 1982, p. 1). De son côté, le Japon réclame à la France de ne pas prendre des mesures protectionnistes et appelle de ses vœux la promotion d'une coopération industrielle entre les deux pays (Le journal du soir Nihon Keizai Shimbun, 17 mars 1982, p. 1). L'article daté du 6 avril 1982 note que le Japon affirme avoir fait une concession sur la réduction tarifaire du cognac et sur la libéralisation des importations du blé, afin de résoudre les conflits commerciaux entre le Japon et la France avant l'arrivée du président Mitterrand (Le journal Nihon Keizai Shimbun, 6 avril 1982, p. 2). L'article daté du 14 avril 1982 souligne que l'arrivée de Mitterrand a accéléré la coopération industrielle et technique entre le Japon et la France: le Japon acceptait l'accord de partenariat avec la France dans le secteur des technologies émergentes comme les robots industriels, la biotechnologie et l'équipement nucléaire (Le journal Nikkei Sangyo Shimbun, 14 avril 1982, p. 1).

Comme nous l'avons vu, l'exposition L'Angélus de Millet : Tendances du Réalisme en France 1848-1870, ouvre le 17 avril, deux jours après l'arrivée du président Mitterrand. On peut penser qu'il a amené ce chef-d'œuvre de Millet, dans un sens symbolique, au public japonais. Cette exposition a attiré 531144 visiteurs, chiffre qui démontre l'engouement du Japon pour L'Angélus.

Rappelons que la France fut à l'initiative de ce prêt au Japon. L'objectif du gouvernement français était de résoudre les conflits commerciaux entre le Japon avant la visite d'État du président Mitterrand. La France a voulu donner l'impression que les tensions majeures entre les deux pays étaient réglées. Elle a voulu profiter d'une visite du président Mitterrand accompagné de L'Angélus pour promouvoir l'expansion des exportations françaises au Japon ainsi que pour améliorer la relation entre les deux pays. Lors de la prononciation du discours du président Mitterrand à la Diète, le parlement japonais, Mitterrand s'est montré compréhensif à l'égard de l'excédent commercial du Japon avec la France: "De quoi l'Europe accuserait-elle le Japon? De son travail, de ses sacrifices, de ses réussites. Cela serait injuste et coupable... II convient de faire de la paix et de l'harmonie un objet de vénération »(AFJ 2018). On sait bien, en général, que la relation entre États dépend beaucoup de ses échanges économiques. Et étant donné que chaque pays essaie de poursuivre l'intérêt national sur la scène diplomatique, les négociations diplomatiques relèvent souvent d'équilibres fragiles. On constate que L'Angélus a été utilisé comme un 
atout dans la mesure où les chefs-d'œuvre peuvent nourrir ce qu'on appelle le soft-power d'un pays et intervenir sur le plan économique ${ }^{6}$.

\section{Le patrimoine comme une contrepartie ou comme une monnaie d'échange}

Nous avons donc démontré, dans cette première partie, que les prêts des chefs-d'œuvre tels que La Vénus de Milo en 1964 et L'Angélus de Millet en 1982, ont un impact économique. Cette deuxième partie abordera le prêt des œuvres et des expositions en tant que monnaie d'échange entre les pays. Commençons par le prêt de La Joconde au Japon en 1974.

\subsection{Le prêt de La Joconde au Japon en 1974}

La Joconde, considérée comme le tableau le plus célèbre du monde, a été prêtée aux ÉtatsUnis en 19637. Ce chef-d'œuvre mondialement connu, associé comme il l'est au Louvre au point de s'y confondre, a aussi voyagé au Japon en 1974. Il a été présenté au Musée national de Tokyo du 20 avril au 10 juin.

L'accord du prêt de La Joconde au Japon a nécessité de longues années avant de se concrétiser. Après la présentation aux États-Unis, elle n'a jamais quitté le Louvre. Au début de l'année 1971, des entreprises privées comme le journal Sankei Shimbun et le groupe de médias Fuji télévison ont mis en place un comité de préparation pour mener à bien ce projet consistant à présenter La Joconde au public japonais (BUNKACHÔ 1975, p. 5). De son côté, le gouvernement français précise, à l'occasion du comité culturel entre le Japon et la France en juin 1972, que le prêt de La Joconde doit être organisé par le gouvernement japonais qui en prendrait toutes les responsabilités (BUNKACHÔ 1975, p. 5). Ce projet ambitieux s'est donc décidé à l'échelle gouvernementale. Le commissaire de l'Agence pour les Affaires Culturelles du Japon a adressé une lettre datée du 19 avril 1973 au Vice-Ministre des affaires étrangères afin que ce dernier prenne l'initiative d'une négociation avec le gouvernement français (BUNKACHÔ 1975, p. 65). Après cela, le gouvernement japonais renouvela à

\footnotetext{
${ }^{6}$ Cependant, il faut noter la nouvelle bataille de Poitiers : le 22 octobre 1982, le ministre du Budget, Laurent Fabius, signe un arrêté pour contraindre les importateurs à ne plus dédouaner leurs magnétoscopes dans les ports mais au centre de la France, à Poitiers. Cet arrêté a pour but de protéger le secteur de l'industrie de l'électronique française contre les produits japonais. Cette mesure est finalement annulée à la fin d'avril 1983. Voir : FARGUES Hélène, "Le 22 octobre 1982, Laurent Fabius met en place le blocus des magnétoscopes japonais", La Croix [en ligne], 11 avril 2012. Disponible sur https://www.la-croix.com/Actualite/EconomieEntreprises/Economie/Le-22-octobre-1982-Laurent-Fabius-met-en-place-le-blocus-des-magnetoscopesjaponais- NG -2012-04-11-791883 (consulté le 4 août 2021). Voir aussi l'article paru dans l'Économiste [en ligne], 25 novembre 2015. Disponible sur https://www.leconomiste.eu/decryptage-economie/368-laurentfabius-contre-les-magnetoscopes-japonais.html (consulté le 4 août 2021).

7 L'histoire du prêt d'œuvres entre la France et les États-Unis est intéressante. Abordons ce sujet à une autre occasion, puisque cet article se concentre sur le déplacement des œuvres entre le Japon et la France.
} 
plusieurs reprises sa demande auprès du gouvernement français pour emprunter La Joconde.

Le 28 septembre 1973, I'accord sur le prêt de La Joconde au Japon est finalement conclu à Paris entre le gouvernement japonais et le gouvernement français. Cet accord a été signé lors du premier entretien entre le premier ministre japonais Kakuei Tanaka et le président de la République Georges Pompidou. Le président Pompidou a donné son accord à condition que le gouvernement japonais prenne la responsabilité de l'assurance du transport ainsi que celle de l'organisation de l'exposition (BUNKACHÔ 1975, p. 5).

Sur le plan budgétaire, ce prêt a été coûteux: le gouvernement japonais a financé l'ensemble des frais à l'exception des frais d'emballage et de transport entre le Musée du Louvre et l'aéroport d'Orly, pris en charge par la France (BUNKACHÔ 1975, p. 8). Il est à noter que la question de l'assurance a été traitée au cours d'un difficile processus de négociation. La Joconde se devait d'être retournée au Louvre de façon sûre et sans détériorations. Quand elle a été prêtée aux États-Unis en 1963, le président de la République française Charles de Gaulle avait confié La Joconde au président américain John F. Kennedy sous la forme d'une indemnisation entre États (BUNKACHÔ 1975, p. 10). En revanche, dans le cas du prêt de La Joconde au Japon en 1974, ce sont des sociétés privées d'assurances qui ont couvert tous les risques. Le gouvernement japonais a conclu un contrat avec une vingtaine d'assurances privées françaises et la Réunion des musées nationaux fut le bénéficiaire de ce contrat (BUNKACHÔ 1975, p. 10).

La Joconde est finalement arrivée au Japon le 17 avril 1974. Elle a attiré 1493781 visiteurs pendant quarante-huit jours. Si l'on observe le nombre total des visiteurs, l'exposition de La Joconde se situe entre l'exposition de La Vénus de Milo en 1964 (1 723476 visiteurs) et celle intitulée Art français en 1961 (1 477388 visiteurs). Au total, 313504 catalogues ont été vendus.

Les raisons pour lesquelles le Japon a souhaité emprunter La Joconde renvoient à leur volonté d'améliorer l'offre artistique auprès du public japonais par la présentation d'un tel chef-d'œuvre, comme le souligne le Premier ministre japonais Kakuei Tanaka dans le catalogue de cette exposition: «Le prêt de la Joconde apportera au public japonais une compréhension approfondie de la culture européenne, une tendance à aimer l'art et un respect profond pour la culture et l'art »(BUNKACHÔ 1975, p. 67, document 11). Néanmoins, ce qui justifie ce prêt est aussi à chercher du côté d'une volonté de développer les échanges culturels et les relations amicales entre le Japon et la France (BUNKACHÔ 1975, p. 67, document 11). 
Pour autant, peut-on en déduire que La Joconde n'a été prêtée au Japon que dans un objectif purement culturel? II est certain que ce prêt s'inscrivait dans une logique d'échange. Cette logique d'échange est attestée par l'envoi du Japon du trésor national Den Tairano Shigemori Zồ à la Fondation Maeght de Saint-Paul-de-Vence pour l'exposition André Malraux en 1973. Quand la Fondation Maeght a souhaité emprunter ce trésor national, le gouvernement japonais a présenté une demande de prêt de La Joconde (BUNKACHÔ 1975, p. 5). On peut dire que La Joconde a été prêtée en contrepartie de ce prêt dans la mesure où les prêts se font sur un principe de réciprocité : une peinture pour une peinture. Mais, les dimensions politiques et économiques sont aussi des volets importants de cet échange.

Comme nous l'avons vu, c'est le président Pompidou qui a autorisé le prêt de La Joconde lors d'une visite en France du Premier ministre japonais Tanaka, en France du 26 au 29 septembre 1973. Cette rencontre avec le président Pompidou ainsi qu'une conférence au sommet du Premier ministre français Pierre Messmer ont permis d'offrir aux différents acteurs l'opportunité d'échanger sur certains problèmes diplomatiques majeurs et, de manière générale, sur les relations franco-japonaises. À cette occasion, le Premier ministre Tanaka a déclaré que le Japon ferait un don d'environ 300 millions de yens aux établissements d'enseignement supérieur français afin de contribuer au progrès de la recherche sur le Japon en France (BUNKACHÔ 1975, p. 65, document 5).

Cette visite officielle du premier ministre Tanaka en France a porté ses fruits en ce qu'elle a permis d'obtenir l'autorisation d'emprunter La Joconde. En revanche, le prêt du chefd'œuvre a occasionné pour le gouvernement japonais des dépenses : le don d'environ 300 millions de yens. On peut donc poser I'hypothèse que La Joconde a servi de monnaie d'échange. Elle a servi d'opérateur, en jouant un rôle majeur dans la politique internationale, en établissant une relation amicale et de confiance entre les deux pays. Georges Pompidou souligne ainsi dans le catalogue de l'exposition : "Elle nous fera, pardelà les mers, mieux nous comprendre et mieux nous aimer " (BUNKACHÔ 1975, p. 67, document 10$)^{9}$.

\subsection{L'exposition de Tôshôdaiji à Paris en 1977}

L'étude portant sur les échanges culturels entre pays demande à identifier la nature de la réciprocité, par l'usage de la logique de «don » et de "contre-don » défini par Maurice Godelier (GODELIER 1996, p. 15). Dans les lignes ci-dessus, nous avons analysé les modalités du prêt effectué par l'institution muséale française et, plus largement, par le gouvernement

\footnotetext{
${ }^{8}$ Ce trésor national désigné en 1951 a été fait un dépôt par Jingoji au Musée national de Kyoto.

${ }^{9}$ Malheureusement, le président Pompidou est décédé le 2 avril 1974, juste avant cette exposition. Ce message fut donc écrit de son vivant. Il avait projeté de faire une visite officielle au Japon avec La Joconde.
} 
français. Les prochaines lignes de cet article seront donc consacrées au prêt octroyé par le Japon à la France.

L'exposition intitulée Tôshôdaiji, Trésors d'un temple japonais a été présentée au Petit Palais du 6 avril au 22 mai 1977. Cette exposition a rencontré un vif succès. Les français ont eu l'occasion de découvrir des trésors du Tôshôdaiji ${ }^{10}$. Ce n'est pas la première fois que des expositions sur l'art japonais sont accueillies à Paris, comme en témoignent les expositions suivantes : L'art japonais à travers les siècles, Musée d'Art moderne, 1958; 150 ans de Peinture au Japon, de Gyokudo à Tessaï, Petit Palais, 1962 ; L'Au-delà dans I'art japonais, Petit Palais, 1963 ; Trésors de la peinture japonais, Musée du Louvre, 1966 (cat. exp. 1977). En parallèle, le Japon a reçu plusieurs expositions sur l'art français telles que : Art français contemporain, œuvres présentées à Paris au Salon de Mai 1950 à Tokyo, 1951 ; Art français au Japon, Tokyo, Kyoto et Fukuoka, 1954 ; Art français 1840-1940 à Tokyo, 1961.

L'exposition du temple bouddhiste Tôshôdaiji a été organisée par la Fondation du Japon en collaboration avec le gouvernement japonais. À l'origine, cette exposition avait été planifiée pour marquer la visite officielle du président de la République française Valéry Giscard d'Estaing au Japon, visite prévue au printemps 1977. Le succès de l'exposition Kaii Higashiyama ${ }^{11}$ à la galerie Yoshii à Paris en 1976 a dû encourager la mise en place de cette exposition (KOKUSAI-KORYU-KIKIN 1977, p. 2). L'exposition Kaii Higashiyama avait en effet présenté les esquisses des peintures sur Fusuma dans Mieidô ${ }^{12}$ du temple Tôshôdaiji. Le directeur général de la Fondation du Japon a donc tenté de montrer les peintures originales sur Fusuma dans les conditions dans lesquelles elles sont montrées au Japon. Pour cela, il a fallu reconstruire la maquette de Mieidô.

Un des éléments notables de cette exposition a été l'acte d'envoyer pour cette occasion la statue représentant le moine Ganjin. Ce remarquable portrait sculpté a été désigné en 1951 "Trésor national ». II n'a jamais quitté son sanctuaire de Tôshôdaiji. De plus, cette statue de Ganjin appartient à ce qu'on appelle en japonais Hibutsu. Hibutsu sont des statues et des tableaux dont les caractères sacrés sont uniquement dédiés aux petits sanctuaires. Ils ne sont jamais exposés au public mais peuvent parfois être montrés pour une occasion spéciale. Dans le cas de la statue de Ganjin, il est permis de la voir une fois par an, entre le 5 et le 7 juin.

On imagine alors aisément comment il a été difficile de faire sortir cette statue du Japon pour l'amener jusqu'en France, accompagnée d'autres sculptures et peintures précieuses

\footnotetext{
10 Tôshôdaiji est un temple bouddhiste qui se situe dans la ville de Nara (Nara fut la première métropole du Japon). Ganjin, moine chinois, a fondé ce temple en 759.

${ }^{11}$ Kaii Higashiyama (1908-1999) est un peintre japonais.

12 Mieidô est un bâtiment dans lequel la statue de Ganjin est placée.
} 
conservées au Tôshôdaiji. En premier lieu, la statue de Ganjin, sculptée au VIII siècle, est extrêmement fragile. Elle ne supporte ni un long voyage, ni un climat différent. En seconde lieu, étant donné que cette statue est Hibutsu, la présentation au public est très limitée. Enfin, comme le propriétaire de cette statue n'est pas un musée, les choses ne se passent donc pas selon la logique propre aux institutions culturelles. Néanmoins, le Grand-Prêtre de Tôshôdaiji a accepté de plein gré d'envoyer cette statue en France : «Ce n'est pas une idée excentrique... La décision selon laquelle Ganjin vient au Japon lui avait pris douze ans et lorsqu'il y est finalement arrivé, il a perdu la vue... Je suis d'accord pour envoyer cette statue à Paris» (Le journal Nihon Keizai Shimbun, 20 mai 1977, in KOKUSAI-KORYU-KIKIN Nihongo Kyoiku Kiyo 1977, n. p.)

L'autorisation de prêter le portrait sculpté de Ganjin, ce trésor national japonais, a été donnée par le Comité de l'examen des experts pour la culture (sous-comité chargé du secteur sculpture) le 4 février 1977 (cat. exp. 1977). Voici les raisons qui ont conduit à la réalisation des deux prêts exceptionnels autorisés par la France : la présentation de La Vénus de Milo au Japon en 1964 et celle de La Joconde en 1974. Comme le souligne un texte du catalogue de cette exposition, «Le Japon a ainsi voulu répondre au prêt prestigieux de La Vénus de Milo et de La Joconde qui lui furent consentis par la France il y a quelques années » (cat. exp. 1977). Le directeur général de la Fondation du Japon a expliqué pourquoi le Japon a décidé d'envoyer le portrait sculpté de Ganjin : «Nous avons pensé que l'envoi de la statue de Ganjin est une réponse appropriée à la décision du gouvernement français de montrer au public japonais les plus importants chefs-d'œuvre comme La Vénus de Milo et La Joconde » (Le journal du soir Tokushima Shimbun, 9 mars 1977, p. 8).

Par ailleurs, il faut rappeler l'influence d'André Malraux. En tant qu'admirateur et sympathisant de la culture japonaise, il a joué un rôle important dans le contexte de l'échange culturel entre les deux pays. Dans un article, il a en effet fait l'éloge de cette statue et a souhaité la montrer au public français (KON 1977, p. 43). Le directeur général de la Fondation du Japon a expliqué comment une telle décision fut prise: "J'ai demandé à Malraux d'emprunter La Joconde afin de la montrer au public japonais il y a dix ans, et mon vœu a été comblé sept ans après. Pour répondre à cette bonne volonté, j'ai choisi d'envoyer Ganjin à Paris. Je suis ravi que les Français aient apprécié la statue de Ganjin et les autres œuvres exposées dans l'exposition de Tôshôdaiji, mais en même temps, je suis triste que Malraux soit décédé. S'il était encore vivant, sans doute aurait-il aimé voir Ganjin à Paris plus que personne d'autre »(KON 1977, p. 43). Ajoutons que le Grand-Prêtre de Tôshôdaiji en personne a répondu à la question posée par la presse française portant sur la raison pour laquelle il a donné la permission d'exposer la statue de Ganjin pendant plus que quarante jours: "Je voudrais vérifier moi-même combien de parisiens apprécient cette statue comme Malraux l'a fait » (Le journal du soir Mainichi Shimbun, 6 mai 1977, p. 5). 
On constate que le prêt de Ganjin effectué par le gouvernement japonais a été réalisé pour répondre aux prêts du gouvernement français qui a confié au Japon les trésors que sont $L a$ vénus de Milo (1964) et La Joconde (1974). Ces chefs-d'œuvre mondialement connus servent de monnaie d'échange. Certes, ce prêt de Ganjin a été fait dans le cadre d'échange culturel entre le Japon et la France, mais pour le Japon, il faut aussi considérer l'existence d'une sorte de pression psychologique : les Japonais s'inquiétaient de ne pas accomplir un prêt d'œuvres équivalent aux prêts que leur avait octroyé la France (Le jounral Yomiuri Shimbun, 6 avril 1977, p. 22).

Or, le prêt de la statue de Ganjin peut soulever une autre question : I'accessibilité aux trésors nationaux ou Hibutsu. Cet événement nous a montré la difficulté d'envoyer ce type d'objet sacré à l'étranger pour une exposition. À partir du moment où la statue de Ganjin a été autorisée à sortir, autorisation octroyée par le comité de l'examen des experts pour la culture le 4 février 1977, on a réfléchi à la possibilité, à l'égard de Tôshôdaiji, d'être présentée davantage au public, décision qui par la suite a été adoptée par ce comité (KOKUSAI-KORYU-KIKIN 1977, p. 3). Pourtant, aujourd'hui même, cette statue n'apparaît que trois jours par an devant le public. Cela nous amène à nous interroger sur l'accès public des trésors nationaux, y compris Hibutsu.

\section{L'inscription du déplacement du patrimoine dans la diplomatie culturelle}

La première partie de cet article fut consacrée au prêt des œuvres, en tenant compte de la dimension économique. La deuxième partie s'est concentrée sur la question du prêt des œuvres et des expositions, en observant la contrepartie à ces prêts qui peuvent être considérés comme monnaie d'échange. Dans cette dernière partie, nous nous interrogerons sur la manière dont le déplacement du patrimoine s'inscrit dans la diplomatie culturelle.

\subsection{Les Saisons culturelles entre pays : l'échange de trésors nationaux entre le Japon et la France}

La France favorise l'action culturelle extérieure à l'État avec un but précis. II s'agit de promouvoir « le rayonnement de la culture française et la mise en œuvre de la diversité culturelle »(MEAE 2015). La France, depuis 1985, organise des programmations culturelles sous trois formes : les Saisons (de 3 à 6 mois), les Années (de 8 à 12 mois) et les Festivals (de 1 à 3 mois). Ces événements culturels sont organisés par le ministère des Affaires étrangères et le ministère de la Culture et mis en œuvre par l'Institut français (MC s. d.). À l'origine, ces événements étaient centrés sur les arts et la culture, mais aujourd'hui, ils s'ouvrent à la recherche, à l'enseignement supérieur, à l'éducation, au sport, au tourisme, à 
l'économie et à la gastronomie (IF s. d.). Quand on étudie l'histoire des Saisons, on relève que plus de cinquante Saisons et Années ont été organisées entre 1985 et 2019. Commencées par l'Année d'Inde en 1985, ces Saisons ou Années sont marquées par des échanges culturels internationaux. Elles donnent lieu à des échanges dans de multiples domaines.

L'Année croisée entre le Japon et la France a été organisée ainsi : I'Année du Japon en France entre 1997 et 1998, et l'Année de la France au Japon entre 1998 et 1999. II est intéressant de remarquer que ces événements mettent en jeu des échanges de trésors nationaux entre les deux pays. La décision de ces échanges a été prise dans le cadre du plan d'action franco-japonais intitulé « 20 actions pour l'an 2000 ». II a été signé par le président de la République Jacques Chirac et le Premier ministre Ryutaro Hashimoto le 18 novembre 1996, lors de la visite d'État du président de la République française au Japon entre le 17 et le 20 novembre.

L'objectif de ce plan d'action était de renforcer la concertation politique et économique et de développer la coopération entre les deux pays (AFJ 2018). Ce plan d'action a été divisé en trois volets : des rencontres régulières à plusieurs niveaux, notamment un sommet annuel franco-japonais, des rencontres bi-annuelles entre les ministres des Affaires étrangères et des entretiens réguliers entre les ministres de l'Économie, enfin une coopération bilatérale approfondie et une coopération sur les grandes questions internationales (SENAT s. d.).

Que signifie ce plan d'action? On peut voir la mise en œuvre de l'organisation de la politique étrangère de la France dans les années 1990. Rappelons que la relation diplomatique entre les deux pays était difficile malgré la visite d'État du président de la République François Mitterrand en 1982. Jacques Chirac est élu président de la République française en mai 1995. Sous sa présidence, la France a repris ses essais nucléaires dans le Pacifique en juin 199513, entraînant la détérioration de la relation franco-japonaise. À l'occasion de la signature du plan d'action franco-japonais le 18 novembre 1996, la France a fait état de deux intentions dans sa diplomatie internationale : d'une part, rétablir la relation avec le Japon, et d'autre part, étendre son influence en Asie, en gardant une position forte au Japon. Par ailleurs, c'est une histoire connue, Jacques Chirac connaît parfaitement la culture japonaise. II a visité le Japon quarante-deux fois avant d'être intronisé président de la République française.

Nous allons voir maintenant comment l'échange de trésors nationaux entre le Japon et la France a été décidé dans le cadre de l'Année croisée entre deux pays. Comme nous l'avons

\footnotetext{
13 Elle les a arrêtées en janvier 1996.
} 
vu, la décision est prise dans le plan d'action « 20 actions pour l'an 2000 ». L'action 10 de ce plan indique que l'Année du Japon en France entre 1997 et 1998 ainsi que l'Année de la France au Japon entre 1998 et 1999 seront l'occasion de promouvoir une compréhension mutuelle entre les deux peuples (MOFA s. d.). C'est à travers l'action 10 que les deux gouvernements ont décidé l'échange de trésors nationaux.

Le choix d'une œuvre échangée n'était pas chose aisée pour ces deux pays. Il a fallu répondre aux trois conditions suivantes : une œuvre doit être classée "trésor national ", une œuvre doit être représentative d'un pays d'un point de vue historique et, en principe, une œuvre ne doit jamais avoir été exposée à l'étranger (FUNATO 1997, p. 6). Or, au Japon, et ceci constitue une difficulté, il n'existe pas beaucoup de trésors nationaux qui puissent satisfaire à ces trois conditions. La plupart des trésors nationaux sont conservés dans des sites sacrés que sont les temples et ne remplissent pas l'ensemble de ces conditions.

En ce qui concerne le choix d'une œuvre, le gouvernement japonais a finalement donné la priorité à l'intention du président de la République Jacques Chirac, qui possède une connaissance approfondie de la culture japonaise (Le journal du soir Yomiuri Shimbun, 4 janvier 1997, p. 1). Chirac a dressé une liste d'œuvres qu'il a voulu faire venir en France. II a noté dans cette liste la statue Kudara Kannon provenant du sanctuaire Hôryûji1 ${ }^{14}$, qui a pu être exposée en France. Kudara Kannon est une statue en bois du Bouddha sculptée au VII siècle (durant l'époque d'Asuka 592-710) Elle est désignée trésor national en 1951 et est conservée à Hôryûji.

En effet, le souhait de présenter Kudara Kannon en France avait déjà été exprimé lorsque La Vénus de Milo fut présentée au Japon en 1964. D'ailleurs, cette statue était appréciée par André Malraux (cat. exp. 1997, p. 29). Le président de la République Jacques Chirac a également mentionné, dans le catalogue de l'exposition, que « La présence à Paris de la Kudara Kannon répond à l'envoi au Japon, il y a trentaine d'années, de La Joconde et de La Vénus de Milo. Ce dialogue se poursuit dans la générosité et l'esprit des échanges culturels tels qu'André Malraux les appelait de ses vœux, favorisant la redécouverte des arts des civilisations lointaines et la valeur universelle de l'œuvre » (cat. exp. 1997, p. 7). On constate que le prêt d'œuvre comme La Vénus de Milo a eu un impact certain dans le contexte des échanges culturels entre les pays.

Par ailleurs, le gouvernement français a choisi La Liberté guidant le peuple d'Eugène Delacroix en tant qu'œuvre échangée. Le Japon a souhaité emprunter ce chef-d'œuvre qui représente la Révolution de Juillet et qui s'appuie sur un fait historique important dans

\footnotetext{
${ }^{14}$ Le monastère Hôryûji se situe dans la région d'Ikaruga de la préfecture de Nara. Étant établit dans le septième siècle, il est connu pour être le plus ancien édifice construit en bois dans le monde. Il fut inscrit au patrimoine mondial de l'humanité de I'UNESCO, une première pour le Japon.
} 
I'histoire française. Pour la France, elle est non seulement l'une des œuvres les plus importantes dans la collection du Musée du Louvre, mais aussi elle est devenue la représentation de la démocratie et le symbole de la République française (cat. exp. 1999, p. 10-11). Cependant, cette œuvre ne répond pas exactement à la troisième condition : elle a été exposée, notamment aux États-Unis, en 1974.

L'exposition intitulée Kudara Kannon, Une sculpture du Japon ancien a été montrée au musée du Louvre du 10 septembre au 13 octobre 1997 dans le cadre de l'Année du Japon en France. Alors que l'exposition Delacroix : la liberté guidant le peuple a été présentée au Musée national de Tokyo du 26 février au 28 mars 1999. Sur la proposition de l'ancien Premier ministre Ryutaro Hashimoto, les peintures japonaises de la première moitié du XIXe siècle ont été exposées dans l'autre salle afin qu'on puisse comparer l'histoire de l'art française avec celle du Japon. Cette exposition a attiré 376581 visiteurs.

Comme nous l'avons vu, la France a pour but de promouvoir l'action culturelle extérieure et les Saisons culturelles entre pays sont une invention française. Quand l'organisation de l'Année croisée entre le Japon et la France a été décidée, cette décision s'est accompagnée d'un grand projet : l'échange de trésors nationaux entre le Japon et la France. Ce projet inédit a été réalisé sur base d'une décision gouvernementale, c'est-à-dire dans le cadre du plan d'action franco-japonais «20 actions pour l'an 2000 ». Ce fait révèle que le déplacement du patrimoine, comme le montre l'étude du cas d'échange de trésors nationaux entre deux pays, s'inscrit au titre de la diplomatie culturelle.

\section{Conclusion}

La politique du prêt, en faisant déplacer des œuvres et des expositions d'un pays à l'autre, est une opération qui peut s'analyser sous l'angle de la diplomatie culturelle. Dans cette perspective, le déplacement des œuvres patrimoniales s'envisage non seulement dans une dimension culturelle, mais aussi selon une dimension politique et économique.

Cet article s'est attaché à présenter les prêts remarquables entre le Japon et la France qui ont marqué l'histoire de la muséologie comme le montre le cas du prêt de La Vénus de Milo au Japon en 1964. Ainsi, nous avons traité la question de la stratégie de la France en matière d'utilisation de son patrimoine dans le contexte des relations internationales, comme l'a montré l'exemple de la relation avec le Japon. Quand La Joconde a été envoyée au Japon en 1974, ce prêt a été réalisé dans une logique d'échange, dans la mesure où les prêts suscitent à leur tour d'autres prêts. Cependant, ce prêt a apporté à la France un don de 300 millions de yens, conférant à La Joconde le rôle de monnaie d'échange. D'ailleurs, quand le Japon a prêté son trésor national, la statue de Ganjin, à la France dans le cadre de l'exposition de Tôshôdaiji en 1977, le Japon, avec ce prêt extraordinaire, a voulu répondre 
à la France qui lui avait confié ses deux chefs-d'œuvre, La Vénus de Milo en 1964 et La Joconde en 1974. On constate que ces chefs-d'œuvre ont rendu possible la présentation en France de la statue de Ganjin qui n'était jusqu'alors jamais sortie de son sanctuaire.

Le prêt des œuvres et des expositions s'effectue dans une logique d'échange, mais comporte aussi un aspect économique comme le montre le cas du prêt de La Vénus de Milo en 1964 et le cas de l'exposition L'Angélus de Millet: Tendances du Réalisme en France 1848-1870 présentée en 1982. Par ailleurs, quand le Japon et la France ont décidé d'échanger leurs trésors nationaux dans le cadre de l'Année croisée entre ces deux pays durant les années 1997 et 1999, cette décision a été élaborée pour accompagner certaines politiques et économiques entre les pays. Comme le montre l'échange des trésors nationaux entre le Japon et la France, la France a fait usage de ses œuvres patrimoniales pour l'exercice de son action politique extérieure à la fois sur le plan culturel et diplomatique.

\section{Liste d'abréviations}

AFJ: Ambassade de France au Japon

AMN : Archives des musées nationaux, service des expositions de la Réunion des musées nationaux

Doc. AGER : Documentation AGER, Musée du Louvre

IF : Institut français

GEFJ : Groupe économique franco-japonais

MC: Ministère de la culture

MEAE: Ministère de l'Europe et des Affaires étrangères

MOFA : Ministère des Affaires étrangères du Japon

\section{Bibliographie}

Archives

ARCHIVES DES MUSEeS NATIONAUX, Pierrefitte-sur-Seine, Service des expositions de la Réunion des musées nationaux, 4CC49.

Documentation AGER, Paris, Musée du Louvre, Ma0399.

Livres

BUNKACHÔ, 1975 : Mona Lisa Ten Kiroku, Tokyo, Agence japonaise des affaires culturelles.

GOdelier Maurice, 1996 : L'énigme du don, Paris, Fayard. 
KOKUSAI-KORYU-KIKIN Nihongo Kyoiku Kiyo, 1977 : Pari Tôshôdaiji Ten sono Kiroku to Hankyô, Tokyo, Fondation du Japon.

Articles parus dans une revue périodique

FUnATO Teruhisa, 1997 : «Nichifutsu Kokuhôkyû Bijutsuhin Kôkantenji ni tsuite », Gekkan-Bunkazai, $n^{\circ} 410$, novembre, p. 4-7.

Kon Hidemi, 1977 : «Pari no Tôshôdaiji Ten », Chuô-Kôron, juin, p. 42-43.

\section{Articles parus dans un journal}

Tokushima Shimbun (du soir), le 9 mars 1977, p. 8.

Yomiuri Shimbun, le 6 avril 1977, p. 22.

Mainichi Shimbun (du soir), le 6 mai 1977, p. 5.

Nihon Keizai Shimbun (du soir), le 17 mars 1982, p. 1.

Nihon Keizai Shimbun, le 6 avril 1982, p. 2.

Nikkei Sangyo Shimbun, le 14 avril 1982, p. 1.

Yomiuri Shimbun (du soir), le 4 janvier 1997, p. 1.

\section{Catalogues d'exposition}

Cat. exp. 1977 : Toshodaiji, Trésors d'un temple japonais, exposition présentée au Petit Palais du 6 avril au 22 mai 1977, Paris, Association Française d'action artistique.

Cat. exp. 1982 : L'Angélus de Millet: Tendances du Réalisme en France 1848-1870, exposition présentée au Musée national d'art Occidental du 17 avril au 13 juin 1982, Tokyo, Yomiuri Shimbun.

Cat. exp. 1999 : Delacroix : la liberté guidant le peuple, exposition présentée au National Museum de Tokyo du 26 février au 28 mars 1999, Tokyo, National Museum de Tokyo.

\section{Documents numériques}

Ambassade de France AU JAPON, 2018 : "Quelques dates clés des relations franco-japonaises», Ambassade de France, Ambassade de France au Japon [en ligne]. Disponible sur https://jp.ambafrance.org/Quelques-dates-cles-des-relations-franco-japonaises (consulté le 4 août 2021).

Groupe ÉCONOMiQue franco-Japonals, s. d.: "Paris Club 1993-2003», Paris Club [en ligne]. Disponible sur http://www.parisclub.gr.jp/wp-content/uploads/2013/08/10 04.pdf (consulté le 3 août 2021).

INSTITUT FRANÇAIS, s. d. : « Les Saisons et Années croisées », Institut français [en ligne]. Disponible sur https://www.pro.institutfrancais.com/fr/offre/presentation-des-saisons-et-annees-croisees (consulté le 4 août 2021). 
Ministere de LA CULtURE, s. d. : "Que sont les Saisons culturelles étrangères en France ", Ministère de la Culture [en ligne]. Disponible sur https://www.culture.gouv.fr/Foire-aux-questions/Que-sont-lesSaisons-culturelles-etrangeres-en-France (consulté le 4 août 2021).

Ministere de l'EUROPE et DES AfFAIRES ETRANGeRES, 2015 : «Diplomatie culturelle », France Diplomatie [en ligne]. Disponible sur https://www.diplomatie.gouv.fr/fr/politique-etrangere-de-lafrance/diplomatie-culturelle/ (consulté le 4 août 2021).

Ministere des Affaires etrangeres du JAPON, s. d. : "20 actions pour l'an 2000 », Mofa [en ligne]. Disponible sur https://www.mofa.go.jp/mofaj/kaidan/yojin/arc 96/france/f sochi.html\#10 (consulté le 4 août 2021).

SenAT, s. d. : « Les Relations Franco-Japonaises », Senat [en ligne]. Disponible sur https://www.senat.fr/ga/ga-028/ga-0283.html (consulté le 4 août 2021).

\section{Notice biographique}

Harumi Kinoshita a obtenu le diplôme de doctorat en Sciences de l'Information et de la Communication à l'Université d'Avignon et des Pays de Vaucluse en 2011 et le diplôme de DEA en Histoire de l'Art à l'Université de Paris 1 en 2005. Ses domaines d'intérêt sont la muséologie et l'histoire de l'art. Elle travaille actuellement en tant que chargée de cours à I'Université Musashi (Tokyo).

Contact : kinoshita.harumi@gmail.com 